\title{
Cognitive impairment and magnetic resonance imaging correlates in primary progressive multiple sclerosis
}

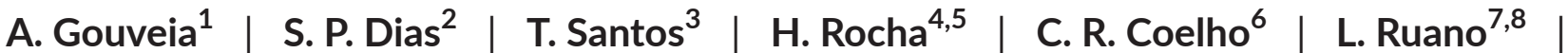 \\ O. Galego $^{9}$ | M. C. Diogo ${ }^{10}$ | D. Seixas ${ }^{11,12}$ | M. J. Sá ${ }^{4,13}$ | S. Batista ${ }^{1,14}$ \\ ${ }^{1}$ Department of Neurology, Centro Hospitalar e Universitário de Coimbra, Coimbra, Portugal \\ ${ }^{2}$ Department of Neurology, Centro Hospitalar de Lisboa Central, Lisboa, Portugal \\ ${ }^{3}$ Department of Neurology, Centro Hospitalar Vila Nova de Gaia/Espinho, Vila Nova de Gaia, Portugal \\ ${ }^{4}$ Department of Neurology, Centro Hospitalar de São João, Porto, Portugal \\ ${ }^{5}$ Faculty of Medicine, Department of Clinical Neuroscience and Mental Health, University of Porto, Porto, Portugal \\ ${ }^{6}$ Department of Neurology, Centro Hospitalar de Setúbal, Setúbal, Portugal \\ ${ }^{7}$ Department of Neurology, Centro Hospitalar Entre Douro e Vouga, Santa Maria da Feira, Portugal \\ ${ }^{8}$ EPIUnit - Epidemiology Research Unit, Institute of Public Health, University of Porto, Porto, Portugal \\ ${ }^{9}$ Department of Neuroradiology, Centro Hospitalar e Universitário de Coimbra, Coimbra, Portugal \\ ${ }^{10}$ Department of Neuroradiology, Centro Hospitalar de Lisboa Central, Lisboa, Portugal \\ ${ }^{11}$ Department of Imaging Diagnosis, Centro Hospitalar de Vila Nova de Gaia/Espinho, Vila Nova de Gaia, Portugal \\ ${ }^{12}$ Faculty of Medicine, Department of Experimental Biology, Porto University, Porto, Portugal \\ ${ }^{13}$ Faculty of Health Sciences, University Fernando Pessoa, Porto, Portugal \\ ${ }^{14}$ Faculty of Medicine, University of Coimbra, Coimbra, Portugal
}

\section{Correspondence}

A. Gouveia, Department of Neurology,

Centro Hospitalar e Universitário de

Coimbra, Praceta Mota Pinto, Coimbra,

Portugal.

Email: anargouveia86@gmail.com

Funding information

Portuguese Multiple Sclerosis Study Group;

Biogen Idec
Objectives: To characterize cognitive impairment in primary progressive multiple sclerosis (PPMS) and to correlate the pattern of cognitive deficits with brain magnetic resonance imaging (MRI) volumetric data.

Materials and methods: In a multicenter cross-sectional study, we recruited consecutive patients with PPMS as well as age, sex, and education level-matched healthy controls (HC). All participants underwent neuropsychological (NP) assessment, and brain MRI was performed in patients with PPMS for analysis of lesion load, subcortical $\mathrm{GM}$ volumes, and regional cortical volumes.

Results: We recruited 55 patients with PPMS and $36 \mathrm{HC}$. Thirty-six patients were included in the MRI analysis. Patients with PPMS performed significantly worse than HC in all NP tests. Subcortical GM volume was significantly correlated with all NP tests, except for Stroop Test, with the largest effect for the thalamus ( $r=-.516$ [BVMT-R DR, $P=.016$ FDR-corrected] to $r=.664$ [SDMT, $P<.001$ FDR-corrected]). In the stepwise linear regression model, thalamic volume was the only predictor of performance in all NP tests.

Conclusion: Cognitive impairment is common in PPMS and affects all evaluated cognitive domains. Subcortical GM volume, particularly of the thalamus, is a strong predictor of cognitive performance, suggesting it has a central role in the pathophysiology of PPMS-related cognitive dysfunction. 


\section{KEYWORDS}

cognitive impairment, magnetic resonance imaging, primary progressive multiple sclerosis, subcortical gray matter, thalamus

\section{1 | INTRODUCTION}

Cognitive impairment has been increasingly recognized as a frequent cause of disability in multiple sclerosis (MS) in all stages of the disease, with a profound impact on activities of daily living and quality of life. ${ }^{1,2}$ It is reported to be present in $15-65 \%$ of patients with $M S^{3,4}$ depending on different cognitive impairment definitions and disease subtypes. ${ }^{1,5,6}$

Due to the relative rarity of primary progressive multiple sclerosis (PPMS), ${ }^{7}$ the impact of cognitive dysfunction on this MS subtype has not yet been fully investigated. The few studies assessing cognitive impairment in PPMS estimate a frequency of cognitive dysfunction ranging from $7 \%$ to $50 \%{ }^{2,4,8}$ and affecting several domains, including attention and working memory, verbal learning, spatial memory, spatial reasoning, and verbal fluency. ${ }^{4,5},-12$ Most of these studies rely on small single-center samples, which may account for the widely varying frequency of cognitive impairment and equivocal results when comparing PPMS with other MS subtypes. In addition, data regarding magnetic resonance imaging (MRI) correlates of cognitive dysfunction in PPMS are scarce. In relapse-onset MS, it is widely recognized that MRI measures of white matter (WM) and gray matter (GM) atrophy are correlated with cognitive dysfunction, generally with GM volume indices accounting for most of the variance. ${ }^{13}$ However, patients with PPMS have MRI characteristics that differ from those with relapse-onset MS, with relatively low burden and activity of MRI-visible lesions on T2weighted and gadolinium-enhanced scans of the brain, ${ }^{14}$ and therefore, these results cannot be directly extrapolated. A few studies have investigated the relationship between MRI findings and cognitive impairment in PPMS. Ukkonen and colleagues ${ }^{15}$ found cognitive deficits in patients with PPMS to be correlated with T1- and T2-lesion load, but not with global brain atrophy, while in a more recent study by Tur et al., GM damage as measured by magnetization transfer ratio was the main correlate of overall cognitive dysfunction. ${ }^{16}$ The influence of individualized subcortical GM structures and regional cortical atrophy on PPMSrelated cognitive impairment, however, remains to be determined.

The purpose of our study was to characterize cognitive impairment in patients with PPMS and to correlate the pattern of cognitive deficits with brain MRI volumetric data.

\section{MATERIALS AND METHODS}

\section{1 | Study population and setting}

In a multicenter, hospital-based, cross-sectional study, we recruited patients with PPMS regularly followed at the Department of Neurology of six Portuguese centers, namely (a) Centro Hospitalar e Universitário de Coimbra (CHUC); (b) Centro Hospitalar de Vila Nova de Gaia/Espinho (CHVNG/E); (c) Centro Hospitalar de São João (CHSJ); (d) Centro Hospitalar de Lisboa Central (CHLC); (e) Centro
Hospitalar de Setúbal (CHS); and (f) Centro Hospitalar de Entre Douro e Vouga (CHEDV).

Patients were consecutively recruited during a six-month period from April to September 2014. Inclusion criteria were as follows: definite diagnosis of PPMS according to the 2010 McDonald criteria ${ }^{17}$; age between 18 and 65; and native Portuguese speaker.

A group of healthy volunteers, matched through age, sex, and education level stratification with patients with PPMS, were recruited and served as healthy controls ( $\mathrm{HC})$.

Exclusion criteria for all participants were a history of neurologic (other than MS in patient group) or systemic disease; a significant visual, auditory, or language impairment that would negatively affect their ability to satisfactorily complete tests or understand test instructions; a history of psychiatric illness, with the exception of stable mild to moderate depressive symptoms; starting or stopping antidepressants in the 2 months prior to the assessment; a history of alcohol, drug, or substance abuse; a history of previous head injury resulting in loss of consciousness; a recent cognitive evaluation (within 1 year); a relapse or steroid treatment within 30 days preceding evaluation.

This study was approved by local ethics committees and by the Portuguese Data Protection Authority, and all participants or their legally authorized representatives gave written informed consent.

\subsection{Clinical assessment}

A full medical history and detailed neurologic examination were obtained for all patients. The following clinical and demographic data were collected: age, years of education, sex, handedness, age of disease onset, age at diagnosis, disease duration, and current diseasemodifying treatment. Physical disability was evaluated using the detailed Kurtzke Expanded Disability Status Scale (EDSS). ${ }^{18}$ For HC, medical history was obtained by interview prior to assessment.

\section{3 | Neuropsychological evaluation}

All participants underwent a neuropsychological (NP) evaluation, which was performed in a single session and using the same test order in all centers. The battery comprised the oral version of the Symbol Digit Modalities Test (SDMT) ${ }^{19,20}$ to evaluate information processing speed; the Brief Visuospatial Memory Test-Revised (BVMT-R) ${ }^{21}$ to assess visuospatial learning and memory; the California Verbal Learning Test (CVLT1) ${ }^{22}$ to evaluate verbal learning and memory; and the Stroop Test ${ }^{23,24}$ for executive function (inhibitory control). This battery is similar to The Brief International Cognitive Assessment for Multiple Sclerosis (BICAMS), ${ }^{25}$ with the addition of the Stroop Test.

Additionally, the Beck Depression Inventory (BDI) ${ }^{26,27}$ and the Modified Fatigue Impact Scale (MFIS) ${ }^{28}$ were used to determine the influence of depression and fatigue, respectively. 


\subsection{MRI acquisition and analysis}

Brain MRI was performed in patients with PPMS, within 3 months of cognitive evaluation. MRI acquisition was performed in five centers using the same acquisition parameters and protocol. MRI scans were analyzed by three neuroradiologists blinded for clinical and NP results.

Patients were examined on 1.5-Tesla MRI scanners (Siemens ${ }^{\circledR}$ Magnetom Avanto, Siemens ${ }^{\circledR}$ Magnetom Symphony and Philips ${ }^{\circledR}$ Inter), using 2-18 coil channels for the acquisition of the following sequences: axial T2-weighted fluid-attenuated inversion recovery (FLAIR) images; axial T2-weighted turbo spin-echo images; axial proton density-weighted images; and three-dimensional (3D) isotropic T1-weighted fast gradient echo images with magnetization-prepared inversion recovery pulse (MP-RAGE). Scans were obtained in an axialoblique orientation, parallel to the subcallosal line.

Global and regional atrophy measures were acquired via brain extraction and tissue segmentation techniques using the 3D MP-RAGE images and the FreeSurfer software v5.3.0 (freely available at http:// surfer.nmr.mgh.harvard.edu/). The process has been described previously ${ }^{29-32}$ and demonstrated good test-retest reliability across scanner manufacturers and field strengths. ${ }^{33}$ Skull-stripping and segmentation were checked and manually corrected whenever necessary. Volumetric data were normalized to the estimated total intracranial volume and included the following individualized structures: total GM, total neocortical GM, total subcortical GM, total WM, frontal cortex, parietal cortex, occipital cortex, temporal cortex, cingulate cortex, subcortical GM nuclei (caudate, putamen, globus pallidus, thalamus, and nucleus accumbens), corpus callosum, lateral ventricles, and III ventricle.

The T1 lesion volumes were obtained with the FreeSurfer software, as described above, using the "T1 white matter hypointensities" volume output. T2 lesion load was measured by experienced observers, who manually contoured the WM lesions slice by slice on the FLAIR acquisition series, using the 3D Slicer software (v4.3.1, freely available at http://www.slicer.org). ${ }^{34}$

\section{5 | Statistical analysis}

Categorical variables are reported as absolute (n) and relative frequencies (\%), and continuous variables are expressed as mean and standard deviation (SD) for variables with a normal distribution, or median and interquartile range (IQR) otherwise. Normality was assessed both visually and through the Kolmogorov-Smirnov test.

Categorical variables were analyzed using the chi-square $(x 2)$ test or the Fisher exact test (as appropriate). An independent-samples $t$ test was used to compare normally distributed continuous variables; for variables with a non-normal distribution, a Mann-Whitney $U$ test was performed. In addition, an analysis of covariance (ANCOVA) was performed adjusted for BDI and MFIS scores for between-group comparison of NP performance.

For each NP score, z-scores were calculated according to the following formula: (patient's score - mean value of $\mathrm{HC}$ group matched for age, sex, and education level)/SD of the matched HC. Impairment in a given NP test was defined as a z-score below the fifth percentile of the matched $\mathrm{HC}$ group.
Pearson's correlation (or Spearman correlation if normality was not assumed) was used to assess the correlation between NP performance, clinical variables, and MRI data, controlled for age and education level.

To account for possible confounders and interactions between variables, a forward stepwise linear regression model (entrance criterion $P<.05$ and exit criterion $P=.10$ ) was generated in order to determine which MRI variables (lesion load and regional volumes) were predictors of cognitive performance on each NP test. In each case, age and education were entered as covariates and retained in block 1. Significant variables in the correlation analysis were entered in block 2 using the forward stepwise technique.

Statistical analyses were performed using IBM SPSS Statistics for Windows, version 22.0 (IBM Corp, Armonk, NY, USA). All tests were two-tailed, with statistical threshold set at $P<.05$ for single tests or at $P<.05$ corrected through false discovery rate (FDR) for multiple testing.

\section{3 | RESULTS}

\section{1 | Demographic and clinical description of population}

Ninety-eight patients with PPMS were identified in the six centers involved in this study. Forty-three were excluded for the following reasons: age over 65 years $^{16}$; refusal to participate ${ }^{12}$; severe dementia ${ }^{5}$; severe physical disability ${ }^{5}$; psychiatric disease ${ }^{3}$; history of head injury resulting in loss of consciousness ${ }^{1}$; and presence of other conditions that could impair cognition. ${ }^{1}$

Fifty-five patients with PPMS (22 from CHUC, six from CHVNG/E, five from CHSJ, 13 from CHLC, four from CHS, and five from CHEDV) and 36 matched HCs were included. Demographic characteristics of all participants and main clinical features of the PPMS group are shown in Table 1.

\subsection{Cognitive performance}

Patients with PPMS performed worse than their matched $\mathrm{HC}$ in all NP tests (Table 2). Regarding depression and fatigue measures, patients with PPMS had significantly higher BDI and MFIS scores than $\mathrm{HC}(P<.001)$.

After controlling for the effect of BDI and MFIS scores using ANCOVA analysis, the differences between patients with PPMS and $\mathrm{HC}$ remained significant in all NP tests except for visual learning (BVMT-R TL): Stroop C: $F(1,87)=5.7, P=.019$; Stroop CW: $F(1,87)=7.7$, $P=.007 ;$ CVLT TL: $F(1,87)=10.2, P=.002$; CVLT DR: $F(1,87)=12.8$, $P=.001$; BVMT-R TL: $F(1,87)=2.9, P=.095$; BVMT-R DR: $F(1,87)=5.9$, $P=.017 ;$ SDMT: $F(1,87)=11.0, P=.001$.

In the group of patients with PPMS, 42 (76.36\%) were impaired in at least one NP test (six [10.90\%] patients failed in one NP test, eight [14.55\%] patients in two NP tests, and 28 [50.90\%] patients in three or more NP tests), while in $\mathrm{HC}$, impairment in one or more NP tests was found in 10 (27.78\%).

Correlation analysis in patients with PPMS, controlled for age and education, did not reveal significant correlations between clinical 
TABLE 1 Demographic characteristics of all participants and clinical features of the PPMS group

\begin{tabular}{|c|c|c|c|}
\hline & $\begin{array}{l}\mathrm{PPMS} \\
\mathrm{N}=55\end{array}$ & $\begin{array}{l}\mathrm{HC} \\
\mathrm{N}=36\end{array}$ & Pvalue \\
\hline $\begin{array}{l}\text { Mean age, years } \\
(\text { mean } \pm \mathrm{SD})\end{array}$ & $52.7 \pm 9.1$ & $51.2 \pm 8.6$ & .433 \\
\hline $\begin{array}{l}\text { Education level, years } \\
\text { (median [IQR]) }\end{array}$ & $9.0(4-17)$ & $9.0(4-18)$ & .720 \\
\hline Female, n (\%) & $31(56.4)$ & $18(50.0)$ & .668 \\
\hline Caucasian, n (\%) & 55 (100.0) & $35(97.2)$ & .396 \\
\hline Right-handed, n (\%) & $54(98.2)$ & $35(97.2)$ & $>.900$ \\
\hline $\begin{array}{l}\text { Age of disease onset, } \\
\text { years (median [IQR]) }\end{array}$ & $42.0(35-53)$ & - & \\
\hline $\begin{array}{l}\text { Disease duration, } \\
\text { years (median [IQR]) }\end{array}$ & $11.0(7-25)$ & - & \\
\hline EDSS (median [IQR]) & $6.0(4-7.5)$ & - & \\
\hline $\begin{array}{l}\text { Patients on disease- } \\
\text { modifying treatment, } \\
\mathrm{n}(\%)\end{array}$ & $33(60)$ & & \\
\hline
\end{tabular}

PPMS, primary progressive multiple sclerosis; HC, healthy controls; EDSS, Kurtzke Expanded Disability Status Scale; SD, standard deviation; IQR, interquartile range.

TABLE 2 Comparison of cognitive performance in patients with PPMS and HC

\begin{tabular}{|c|c|c|c|}
\hline & $\begin{array}{l}\text { PPMS } \\
\mathrm{N}=55\end{array}$ & $\begin{array}{l}\mathrm{HC} \\
\mathrm{N}=36\end{array}$ & Pvalue \\
\hline \multicolumn{4}{|l|}{ Stroop } \\
\hline $\mathrm{C}$ (median [IQR]) & $84.0(69-342)$ & $65.0(56-110)$ & $<.001$ \\
\hline $\begin{array}{l}\text { CW (median } \\
{[\text { IQR]) }}\end{array}$ & $175.0(142-505)$ & $135.0(118-260)$ & $<.001$ \\
\hline $\begin{array}{l}\text { CVLT1 TL } \\
(\text { mean } \pm \text { SD) }\end{array}$ & $40.2(13.4)$ & $52.6(9.6)$ & $<.001$ \\
\hline $\begin{array}{l}\text { CVLT1 DR } \\
(\text { mean } \pm \text { SD) }\end{array}$ & $9.0(3.9)$ & $12.7(1.8)$ & $<.001$ \\
\hline $\begin{array}{l}\text { BVMT-R TL } \\
(\text { mean } \pm \text { SD) }\end{array}$ & $17.6(10.2)$ & $23.1(5.9)$ & .005 \\
\hline $\begin{array}{l}\text { BVMT-R DR } \\
\text { (median [IQR]) }\end{array}$ & $7.0(4-12)$ & $10.0(9-12)$ & .002 \\
\hline $\begin{array}{l}\text { SDMT } \\
\quad(\text { mean } \pm \text { SD) }\end{array}$ & $32.5(16.7)$ & $47.4(12.0)$ & $<.001$ \\
\hline BDI (median [IQR]) & $11.0(6-26)$ & $4.0(0-14)$ & $<.001$ \\
\hline MFIS (mean \pm SD) & $36.5(18.4)$ & 9.7 (19.9) & $<.001$ \\
\hline
\end{tabular}

PPMS, primary progressive multiple sclerosis; $\mathrm{HC}$, healthy controls; Stroop C, Stroop color naming; Stroop CW, Stroop color-word naming; CVLT1 TL, California Verbal Learning Test - first edition, total learning; CVLT1 DR, California Verbal Learning Test - first edition, delayed recall; BVMT-R TL, Brief Visuospatial Memory Test - Revised, Total Learning; BVMT-R DR, Brief Visuospatial Memory Test - Revised, Delayed Recall; SDMT, Symbol Digit Modalities Test; BDI, Beck Depression Inventory; MFIS, Modified Fatigue Impact Scale; SD, standard deviation; IQR, interquartile range. Data are given as raw scores.

$P$ values are corrected for multiple comparisons by false discovery rate method. $P$ values with statistical significance are indicated in bold. variables (including disease duration, age of MS onset, EDSS, BDI, and MFIS scores) and performance on NP tests ( $P>.05$ FDR-corrected). Similarly, sex distribution was not associated with significant differences in all NP tests.

\section{3 | Brain MRI parameters and cognitive performance in patients with PPMS}

Of the 55 patients with PPMS included, 42 underwent brain MRI. In the other 13 patients, MRI could not be performed due to technical limitations, ${ }^{5}$ patient refusal, ${ }^{5}$ and claustrophobia. ${ }^{3}$

The group of patients that was not able to undergo MRI was not significantly different from the remaining sample regarding age, sex distribution, education, disease duration, and EDSS (Table S1).

Brain structures and lesion load volumes are shown in Table 3. Correlations between brain MRI variables and cognitive tests in patients with PPMS, controlled for age and education, are presented in Table 4.

Subcortical GM volume was significantly correlated with the score of all NP tests, with the largest effect for thalamic volume ranging from $r=-.516$ (BVMT-R DR, $P=.016$ FDR-corrected) to $r=.664$ (SDMT, $P<.001$ FDR-corrected). Conversely, total neocortical GM was only modestly correlated with visuospatial learning (BVMT-R TL, $r=.380$, $P=.04$ FDR-corrected).

Regarding WM, total volume was not significantly correlated with any NP test. On the other hand, corpus callosum volume was significantly correlated with the majority of cognitive domains, ranging from $r=-.429$ (BVMT-R TL, $P=.048$ FDR-corrected) to $r=.531$ (SDMT, $P<.001$ FDR-corrected).

The Stroop Test did not correlate significantly with any cortical region or subcortical structure for a statistical threshold at $P<.05$ FDR-corrected and therefore was not analyzed further on regression models.

The regression models predicting performance on NP tests were controlled for the effects of age and education (Table 5). In each case, only those MRI variables that were statistically significant in the correlation analysis were entered into the models. Thalamic volume was the only predictor of performance in all NP tests.

\section{4 | DISCUSSION}

In the present study, we have characterized the cognitive profile of a multicenter cohort of patients with PPMS and compared it with a matched group of HC. Patients with PPMS performed significantly worse than their matched $\mathrm{HC}$ in all evaluated cognitive domains, which is in agreement with previous studies. ${ }^{5,11,15}$

In an attempt to investigate the pathophysiology of cognitive impairment in PPMS, we analyzed the contribution of several brain MRI parameters in this group of patients, including conventional measures of lesion load and WM and GM volumetric measures. To the best of our knowledge, this is the first study assessing the influence of individualized subcortical GM structures and regional cortical atrophy in PPMS-related cognitive impairment. 
TABLE 3 Brain strutures and lesion load mean volumes of patients with PPMS

\begin{tabular}{|llll}
\hline Total neocortical GM & $0.26045 \pm 0.02389$ & Total white matter & $0.28605 \pm 0.02713$ \\
\hline Frontal cortex & $0.04682 \pm 0.00379$ & Corpus Callosum & $0.00146 \pm 0.00051$ \\
\hline Parietal cortex & $0.03009 \pm 0.00400$ & Lateral ventricles & $0.00985 \pm 0.00506$ \\
\hline Temporal cortex & $0.03086 \pm 0.00327$ & III ventricle & $0.00116 \pm 0.00048$ \\
\hline Occipital cortex & $0.01327 \pm 0.00127$ & & \\
\hline Cingulate gyrus & $0.00630 \pm 0.00064$ & & \\
\hline Total subcortical GM & $0.03241 \pm 0.00292$ & & \\
\hline Thalamus & $0.00411 \pm 0.00041$ & & \\
\hline Putamen & $0.00264 \pm 0.00043$ & & $14459.68 \pm 11041.10$ \\
\hline Caudate & $0.00216 \pm 0.00028$ & & $6489.93 \pm 4966.82$ \\
\hline Globus pallidus & $0.00081 \pm 0.00011$ & T2 lesion load & \\
\hline N. accumbens & $0.00024 \pm 0.00006$ & T1 lesion load & \\
\hline
\end{tabular}

PPMS, primary progressive multiple sclerosis; GM, gray matter.

The values shown are the ratio between absolute volumes and total intracranial volume, except for T2 and T1 lesion load $\left(\mathrm{mm}^{3}\right)$. Data are given as mean \pm standard deviation.

TABLE 4 Correlation between MRI parameters and NP performance in patients with PPMS, controlling for age and education

\begin{tabular}{|c|c|c|c|c|c|c|c|}
\hline Volumes & StroopC & Stroop CW & CVLT1 TL & CVLT1 DR & BVMTR TL & BVMTR DR & SDMT \\
\hline T1 lesion load & 0.054 & 0.05 & $-0.385^{*}$ & $-0.402^{*}$ & $-0.425^{* *}$ & $-0.471^{* *}$ & $-0.356^{*}$ \\
\hline Frontal cortex & -0.073 & -0.039 & 0.197 & 0.081 & $0.319^{*}$ & $0.396^{*}$ & 0.189 \\
\hline Parietal cortex & 0.104 & 0.210 & -0.059 & -0.115 & $0.352^{*}$ & 0.283 & 0.073 \\
\hline Occipital cortex & 0.100 & 0.199 & -0.063 & -0.169 & $0.344^{*}$ & $0.383^{*}$ & -0.026 \\
\hline Cingulate gyrus & 0.257 & $0.359^{*}$ & 0.124 & 0.156 & 0.172 & 0.095 & 0.057 \\
\hline Total subcortical GM & -0.357 & -0.363 & 0.450 & 0.455 & $0.407^{*}$ & 0.406 & $0.662^{* * *}$ \\
\hline Thalamus & -0.294 & $-0.329^{*}$ & $0.579^{* * *}$ & $0.525^{* *}$ & $0.560^{* * *}$ & $0.516^{* *}$ & $0.664^{* * *}$ \\
\hline N. accumbens & -0.069 & 0.017 & 0.304 & 0.290 & 0.246 & 0.305 & 0.255 \\
\hline Total White matter & -0.283 & -0.194 & 0.142 & 0.148 & 0.125 & 0.205 & 0.205 \\
\hline Corpus Callosum & -0.253 & -0.336 & $0.475^{*}$ & $0.438^{*}$ & $0.429^{*}$ & 0.400 & $0.531^{* * *}$ \\
\hline
\end{tabular}

Stroop C, Stroop color naming; Stroop CW, Stroop color-word naming; CVLT1 TL, California Verbal Learning Test - first edition, total learning; CVLT1 DR, California Verbal Learning Test - first edition, delayed recall; BVMTR TL, Brief Visuospatial Memory Test - Revised, Total Learning; BVMTR DR, Brief Visuospatial Memory Test - Revised, Delayed Recall; SDMT, Symbol Digit Modalities Test; GM, gray matter.

All correlations are Pearson $r$ coefficients. Statistical significance is indicated in bold. ${ }^{*} P<.05 ;{ }^{* *} P<.01 ;{ }^{* * *} P<.001$ (corrected for multiple testing by false discovery rate method).

The results obtained suggest that GM damage is important in PPMS, appearing as the main contributor to the related cognitive impairment, beyond the influence of WM pathology. On the other hand, it is noteworthy that subcortical GM volumes, particularly of the thalamus, accounted for most of the variance in cognitive tasks performance, even when compared with cortical GM volumes. Executive function performance, measured by the Stroop Test, did not correlate significantly with any cortical region or subcortical structure, probably reflecting that this complex cognitive domain requires a dynamic interplay of several large-scale neural networks, hindering the possibility of capturing it with a pure MRI volumetric approach. Additionally, measurement accuracy is lower for small volumes and may have compromised the assessment of the contribution of individual GM structures to executive functions. In the remaining cognitive domains (episodic verbal learning and memory, information processing speed, and visuospatial learning and memory), thalamic volume emerged as the only independent predictor of dysfunction.

Since the original conceptualization by Alexander et al., ${ }^{35}$ of parallel cortico-basal ganglia-thalamocortical loops connecting the basal ganglia and the thalamus to the frontal cortex, the role of these structures 


\begin{tabular}{lllll} 
& Step $1 \mathbf{R}^{2}$ & $\begin{array}{l}\text { Variables Retained After Step } 1 \\
\text { (Standardized Beta and } \mathbf{R}^{2} \text { change) }\end{array}$ & Final Model $\mathbf{R}^{2}$ & P value \\
\hline CVLT1 TL & .041 & Thalamus $(0.570 ; 0.321)$ & .362 & $<.001$ \\
CVLT1 DR & .019 & Thalamus $(0.523 ; 0.270)$ & .289 & .001 \\
\hline BVMT-R TL & .306 & Thalamus $(0.469 ; 0.217)$ & .523 & $<.001$ \\
BVMT-R DR & .354 & Thalamus $(0.417 ; 0.172)$ & .525 & .001 \\
SDMT & .461 & Thalamus $(0.490 ; 0.237)$ & .699 & $<.001$ \\
\hline
\end{tabular}

TABLE 5 Linear regression models predicting performance in each NP test, controlled for age and education

CVLT1 TL, California Verbal Learning Test - first edition, total learning; CVLT1 DR, California Verbal Learning Test - first edition, delayed recall; BVMT-R TL, Brief Visuospatial Memory Test - Revised, Total Learning; BVMT-R DR, Brief Visuospatial Memory Test - Revised, Delayed Recall; SDMT, Symbol Digit Modalities Test; GM, gray matter.

in cognition has been emphasized. In line with this, previous studies in relapse-onset MS examining the influence of subcortical GM structures on cognition have revealed a strong correlation between these and cognitive performance. ${ }^{13}$ Among subcortical GM structures, the thalamus has shown the strongest correlation with cognitive impairment in MS studies. ${ }^{36}$ In PPMS, it has been reported that, in clinically early disease, atrophy is most prominent in subcortical GM structures, particularly in the thalamus, with cortical and infratentorial atrophy developing later as the disease progresses. ${ }^{37}$ More importantly, it has been demonstrated that thalamic damage independently predicts long-term accumulation of disability in PPMS. ${ }^{38}$ Our study is the first to suggest that thalamic volume is a significant predictor of PPMS-related cognitive impairment. Interestingly, thalamic volume was an independent predictor of verbal and visuospatial memory, which are typically impaired in conditions affecting the mesial temporal cortex. The thalamus, specifically the anterior and dorsomedial nuclei, is part of the Papez circuit and therefore is a critical structure for episodic memory. Recent theories propose a reciprocal connection between the hippocampus and the thalamus, the latter being implicated in executive aspects of episodic memory (selection of to-be-coded information and retrieval strategies), ${ }^{39}$ which could offer some explanation for our data.

There are some limitations to our study to be considered. First of all, it is limited by the cross-sectional design. Second, although the same clinical procedures and MRI acquisition parameters were used in all centers, there could have been some variability that the small sample size could not overcome. Third, the relatively modest sample size may have prevented us from fully examining the influence of other variables on cognitive performance. Moreover, the use of stepwise linear regression procedures has some limitations, particularly the propensity to overfit the data and the fact that the models created may be an over-simplification of the real ones. Finally, we did not assess other MRI variables that may have accounted for variance in cognitive performance, such as GM demyelinating lesions, and measures of diffuse occult disease by MRI spectroscopy, magnetization transfer, and diffusion tensor imaging of normal-appearing brain tissue.

Apart from these concerns, our study furthers the knowledge of cognitive dysfunction in PPMS and its respective underlying mechanisms. We conclude that cognitive impairment is common in PPMS and affects several cognitive domains including information processing speed, visual memory, verbal memory, and executive functions. Subcortical GM volume, particularly of the thalamus, was the strongest predictor of cognitive impairment, suggesting it has a crucial contribution to cognition in PPMS.

\section{ACKNOWLEDGMENTS}

This study was performed as part of the EMIN (Multiple Sclerosis for Neurology Residents) education and research program for young neurologists, sponsored by the Portuguese Multiple Sclerosis Study Group and Biogen Idec. However, the study did not receive any direct funding for its conduction.

\section{CONFLICT OF INTEREST}

On behalf of all authors, the corresponding author states that there is no conflict of interest.

\section{REFERENCES}

1. Amato MP, Zipoli V, Portaccio E. Multiple sclerosis-related cognitive changes: a review of cross-sectional and longitudinal studies. J Neurol Sci. 2006;245:41-46.

2. Wachowius U, Talley M, Silver N, Heinze HJ, Sailer M. Cognitive impairment in primary and secondary progressive multiple sclerosis. J Clin Exp Neuropsychol. 2005;27:65-77.

3. Rao SM, Leo GJ, Ellington L, Nauertz T, Bernardin L, Unverzagt F. Cognitive dysfunction in multiple sclerosis. II. Impact on employment and social functioning. Neurology. 1991;41:692-696.

4. Camp SJ, Stevenson VL, Thompson AJ, et al. Cognitive function in primary progressive and transitional progressive multiple sclerosis: a controlled study with MRI correlates. Brain. 1999;122(Pt 7):1341-1348.

5. Huijbregts SC, Kalkers NF, de Sonneville LM, de Groot V, Reuling $\mathrm{IE}$, Polman $\mathrm{CH}$. Differences in cognitive impairment of relapsing remitting, secondary, and primary progressive MS. Neurology. 2004;63:335-339.

6. Gajofatto A, Turatti M, Bianchi MR, et al. Benign multiple sclerosis: physical and cognitive impairment follow distinct evolutions. Acta Neurol Scand. 2016;133:183-191.

7. Miller DH, Leary SM. Primary-progressive multiple sclerosis. Lancet Neurol. 2007;6:903-912.

8. Rosti-Otajärvi E, Ruutiainen J, Huhtala H, Hämäläinen P. Relationship between subjective and objective cognitive performance in multiple sclerosis. Acta Neurol Scand. 2014;130:319-327. 
9. Foong J, Rozewicz L, Chong WK, Thompson AJ, Miller DH, Ron MA. A comparison of neuropsychological deficits in primary and secondary progressive multiple sclerosis. J Neurol. 2000;247:97-101.

10. De Sonneville LM, Boringa JB, Reuling IE, Lazeron RH, Ader HJ, Polman $\mathrm{CH}$. Information processing characteristics in subtypes of multiple sclerosis. Neuropsychologia. 2002;40:1751-1765.

11. Ruet A, Deloire M, Charre-Morin J, Hamel D, Brochet B. Cognitive impairment differs between primary progressive and relapsing-remitting MS. Neurology. 2013;80:1501-1508.

12. Hanssen KT, Beiske AG, Landro NI, Hessen E. Predictors of executive complaints and executive deficits in multiple sclerosis. Acta Neurol Scand. 2014;129:234-242.

13. Batista S, Zivadinov R, Hoogs $M$, et al. Basal ganglia, thalamus and neocortical atrophy predicting slowed cognitive processing in multiple sclerosis. J Neurol. 2012;259:139-146.

14. Filippi M, Rovaris $M$, Rocca MA. Imaging primary progressive multiple sclerosis: the contribution of structural, metabolic, and functional MRI techniques. Mult Scler. 2004;10(Suppl 1):S36-S44. discussion S-5.

15. Ukkonen M, Vahvelainen T, Hamalainen P, Dastidar P, Elovaara I. Cognitive dysfunction in primary progressive multiple sclerosis: a neuropsychological and MRI study. Mult Scler. 2009;15:1055-1061.

16. Tur C, Penny S, Khaleeli Z, et al. Grey matter damage and overall cognitive impairment in primary progressive multiple sclerosis. Mult Scler, 2011;17:1324-1332.

17. Polman $\mathrm{CH}$, Reingold SC, Banwell $\mathrm{B}$, et al. Diagnostic criteria for multiple sclerosis: 2010 Revisions to the McDonald criteria. Ann Neurol. 2011;69:292-302.

18. Kurtzke JF. Rating neurologic impairment in multiple sclerosis: an expanded disability status scale (EDSS). Neurology. 1983;33:1444-1452.

19. Smith A. Symbol Digit Modalities Test: Manual. Los Angeles, CA Western Psychological Services; 1982.

20. Rao SM.AManual forthe Brief. Repeatable Battery of Neuropsychological Tests in Multiple Sclerosis: National Multiple Sclerosis Society; 1991.

21. Benedict RHB. Brief Visuospatial Memory Test-Revised: Professional Manual. Odessa, FL: Psychological Assessment Resources; 1997.

22. Delis DC, Kramer JH, Kaplan E, Ober BA. The California Verbal Learning Test. San Antonio, TX: Psychological Corporation; 1987.

23. Stroop JR. Studies of interference in serial verbal reactions. J Exp Psychol Gen. 1935;18:643-662.

24. Greshwater G. The Stroop Color and Word Test: A Manual for Clinical and Experimental Uses. Wood Dale, IL: Stoelting Co.; 2002.

25. Langdon D, Amato M, Boringa J, et al. Recommendations for a Brief International Cognitive Assessment for Multiple Sclerosis (BICAMS). Mult Scler 2012;18:891-898.

26. Vaz Serra APA JL. Aferição dos quadros depressivos I - Ensaio de aplicação do Inventário Depressivo de Beck a uma amostra portuguesa de doentes deprimidos. Coimbra Médica. 1973;20:623-644.

27. Vaz Serra A, Pio Abreu JL. Aferição dos quadros depressivos II Estudo preliminar de novos agrupamentos sintomatológicos para complemento do Inventário Depressivo de Beck. Coimbra Médica. 1973;20:713-736.
28. Fisk JD, Pontefract A, Ritvo PG, Archibald CJ, Murray TJ. The impact of fatigue on patients with multiple sclerosis. Can J Neurol Sci. 1994;21:9-14

29. Dale AM, Fischl B, Sereno MI. Cortical surface-based analysis I. Segmentation and surface reconstruction. Neurolmage. 1999;9:179-194

30. Fischl B, Sereno MI, Dale AM. Cortical surface-based analysis. II: inflation, flattening, and a surface-based coordinate system. Neurolmage. 1999;9:195-207.

31. Fischl B, Dale AM. Measuring the thickness of the human cerebral cortex from magnetic resonance images. Proc Natl Acad Sci U S A. 2000;97:11050-11055

32. Fischl B, van der Kouwe A, Destrieux C, et al. Automatically parcellating the human cerebral cortex. Cereb Cortex. 2004;14:11-22.

33. Han X, Jovicich J, Salat D, et al. Reliability of MRI-derived measurements of human cerebral cortical thickness: the effects of field strength, scanner upgrade and manufacturer. Neurolmage. 2006;32:180-194.

34. Cohen AB, Neema M, Arora A, et al. The Relationships among MRIDefined Spinal Cord Involvement, Brain Involvement, and Disability in Multiple Sclerosis. J Neuroimaging. 2012;22:122-128.

35. Alexander GE, DeLong MR, Strick PL. Parallel organization of functionally segregated circuits linking basal ganglia and cortex. Annu Rev Neurosci. 1986;9:357-381

36. Inglese M, Liu S, Babb JS, Mannon LJ, Grossman RI, Gonen O. Threedimensional proton spectroscopy of deep gray matter nuclei in relapsing-remitting MS. Neurology. 2004;63:170-172.

37. Sepulcre J, Sastre-Garriga J, Cercignani M, Ingle GT, Miller DH, Thompson AJ. Regional gray matter atrophy in early primary progressive multiple sclerosis: a voxel-based morphometry study. Arch Neurol. 2006;63:1175-1180.

38. Mesaros S, Rocca MA, Pagani E, et al. Thalamic damage predicts the evolution of primary-progressive multiple sclerosis at 5 years. AJNR Am J Neuroradiol 2011;32:1016-1020.

39. Stewart CC, Griffith HR, Okonkwo OC, et al. Contributions of volumetrics of the hippocampus and thalamus to verbal memory in temporal lobe epilepsy patients. Brain Cogn. 2009;69:65-72.

\section{SUPPORTING INFORMATION}

Additional Supporting Information may be found online in the supporting information tab for this article.

How to cite this article: Gouveia, A., Dias, S. P., Santos, T., Rocha, H., Coelho, C. R., Ruano, L., Galego, O., Diogo, M. C., Seixas, D., Sá, M. J. and Batista, S. Cognitive impairment and MRI correlates in primary progressive multiple sclerosis. Acta Neurol Scand. 2017;136:109-115. https://doi.org/10.1111/ane.12702 